\title{
Shadowable Point for Non-Autonomous Discrete Dynamical Systems
}

\author{
Le Huy Tien* and Le Duc Nhien \\ Department of Mathematics, Mechanics and Informatics, Vietnam National University at Hanoi, 334 Nguyen \\ Trai, Thanh Xuan, Hanoi, Viet Nam \\ Email: tienlh@viasm.edu.vn
}

\begin{abstract}
The purpose of this paper is to study about the set of shadowable points of nonautonomous dynamical systems $\left(X,\left\{f_{n}\right\}_{n \in \mathbb{Z}}\right)$ where $X$ is a metric space and $f_{n}$ is a homeomorphism for all $n \in \mathbb{Z}$. In particular, we would like to show that $X$ is totally disconnected for every shadowable and almost periodic points with a standard condition. Moreover, if $\left\{f_{n}\right\}_{n \in \mathbb{Z}}$ is equi-continuous then we have converse of the previous property.
\end{abstract}

Keywords: Shadowable point, homeomorphism, metric space, non-autonomous systems.

\section{Introduction and Preliminaries}

Shadowing property has been the subject of numerous studies in the qualitative theory of dynamical systems (see [1], [2]). In 2000, Yuan, Yorke defined the concept of absolutely non-shadowable points in [3], it splits the pseudo-orbit tracing property into individual shadowings. From their ideal, recently, Morales (2016) introduced the notion of shadowable points by individualizing the shadowing property into pointwise shadowings. A shadowable point of a continuous map is defined to be a point such that the shadowing lemma holds for pseudo-orbits beginning at the point. After that, Kawaguchi extends the study on shadowable points recently introduced by Morales in relation to chaotic or non-chaotic properties [4]. About the non-autonomous case, Duarte and Klein give the shadowing property for nonautonomous systems which satisfy several conditions of the maps $f_{n}$ and pseudo-orbits in Avalanche principle proof [5]. In this paper, some new concepts are introduced for non-autonomous discrete systems, including shadowable points, totally disconnected property of $X$. Then, we concern sevaral important properties of them. In particular, we show that $X$ is totally disconnected for every shadowable and almost periodic points with a standard condition. If $\left\{f_{n}\right\}_{n \in \mathbb{Z}}$ is equi-continuous then we have converse of previous property.

Now we introduce some basic notations for non-autonomous dynamical systems. Throughout this paper, by a non-autonomous discrete dynamical system, we mean a pair $\left(X,\left\{f_{n}\right\}_{n \in \mathbb{Z}}\right)$ with $X$ is metric space and $\left\{f_{n}\right\}_{n \in \mathbb{Z}} \subset \operatorname{Hom}(X)$ where $\operatorname{Hom}(X)$ the set of homeomorphisms from $X$ to $X$. One denote $\operatorname{Orb}(p, m)=\left\{f_{m}^{n}(p): \forall n \in \mathbb{Z}\right\}$ is the orbit which is through $p$ at the time $m \in \mathbb{Z}$, where

$$
f_{m}^{n}:= \begin{cases}f_{n-1} \circ \cdots \circ f_{m}, & \text { if } n>m \\ f_{n}^{-1} \circ \cdots \circ f_{m-1}^{-1}, & \text { if } n<m \\ i d, & \text { if } n=m .\end{cases}
$$

A sequence $\left\{x_{n}\right\}_{n \in \mathbb{Z}} \subset X$ is called be a $\delta$-pseudo-orbit if $d\left(f_{n}\left(x_{n}\right), x_{n+1}\right) \leqslant \delta$. The next definitions is extend concepts of shadowable points (see [6], [7], [8]). A point $p \in X$ is shadowable at the time $i \in \mathbb{Z}$ if for every $\varepsilon>0$ there is $\delta>0$, which is depend on $i, \varepsilon$ and $p$, such that every $\delta$-pseudo-orbits $\left\{x_{n}\right\}_{n \in \mathbb{Z}}$ with $x_{i}=p$ is $\varepsilon$-shadowed (i.e. there is $y \in X$ such that $d\left(y_{n}, x_{n}\right) \leqslant \varepsilon$ where $y_{n}=f_{i}^{n}(y)$ ). Moreover, we say that a point $p \in X$ is two-sided limit shadowable at the time $i \in \mathbb{Z}$ if $\lim _{n \rightarrow \pm \infty} d\left(f_{i}^{n}\left(x_{n}\right), x_{n+1}\right)=0$, implies that there is $x \in X$ such that $\lim _{n \rightarrow \pm \infty} d\left(f_{i}^{n}(y), x_{n}\right)=0$. The set of shadowable points (resp. twosided limit shadowable) of $\left(X,\left\{f_{n}\right\}_{n \in \mathbb{Z}}\right)$ at the time $i$ is denoted by $\mathcal{S}\left(i,\left\{f_{n}\right\}_{n \in \mathbb{Z}}\right)\left(\right.$ resp. $\mathcal{L S}\left(i,\left\{f_{n}\right\}_{n \in \mathbb{Z}}\right)$ ). The set

$$
\mathcal{S}\left(\left\{f_{n}\right\}_{n \in \mathbb{Z}}\right)=\bigcup_{i \in \mathbb{Z}} \mathcal{S}\left(i,\left\{f_{n}\right\}_{n \in \mathbb{Z}}\right) \quad\left(\operatorname{resp} . \mathcal{L} \mathcal{S}\left(\left\{f_{n}\right\}_{n \in \mathbb{Z}}\right)=\bigcup_{i \in \mathbb{Z}} \mathcal{L} \mathcal{S}\left(i,\left\{f_{n}\right\}_{n \in \mathbb{Z}}\right)\right)
$$


is called the set of shadowable points.

Definition 1.1. Let the dynamical systems $\left(X,\left\{f_{n}\right\}_{n \in \mathbb{Z}}\right)$ where $f_{n}: X \rightarrow X$ is homeomorphism for all $n \in \mathbb{Z}$. For $Y \subset X$, we now define the omega-limit set

$$
\omega\left(Y,\left\{f_{n}\right\}_{n \in \mathbb{Z}}\right):=\left\{q \in X: \lim _{k \rightarrow+\infty} f_{i}^{n_{k}}(p)=q \text { for some sequence } n_{k} \rightarrow+\infty \text { and for some } p \in Y\right\} .
$$

In the invertible case one also define the alpha-limit set

$$
\alpha\left(Y,\left\{f_{n}\right\}_{n \in \mathbb{Z}}\right):=\left\{q \in X: \lim _{k \rightarrow+\infty} f_{i}^{n_{k}}(p)=q \text { for some sequence } n_{k} \rightarrow-\infty \text { and for some } p \in Y\right\} .
$$

Following [9] we say that $p \in X$ is a point with converging semiorbits if both $\alpha\left(p,\left\{f_{n}\right\}_{n \in \mathbb{Z}}\right)$ and $\omega\left(p,\left\{f_{n}\right\}_{n \in \mathbb{Z}}\right)$ reduce to singleton. Denote by $\mathcal{A}\left(\left\{f_{n}\right\}_{n \in \mathbb{Z}}\right)$ the set of points with converging semiorbits and $\mathcal{C}\left(\left\{f_{n}\right\}_{n \in \mathbb{Z}}\right)$ the set of points $p$ in $\mathcal{A}\left(\left\{f_{n}\right\}_{n \in \mathbb{Z}}\right)$ with $\alpha\left(p,\left\{f_{n}\right\}_{n \in \mathbb{Z}}\right)=\omega\left(p,\left\{f_{n}\right\}_{n \in \mathbb{Z}}\right)=\{p\}$. If $f_{n}=$ f for all $n \in \mathbb{Z}$ then we denote $\left\{f_{n}\right\}_{n \in \mathbb{Z}}$ by $f$ for short.

Definition 1.2 (see [10]). The metric space $X$ is totally disconnected at $p$ if the connected component of $X$ containing $p$ is $\{p\}$. We define

$$
X^{\mathrm{deg}}=\{p \in X: X \text { is totally disconnected at } p\} .
$$

Definition 1.3. Assume that $\left\{D_{n}\right\}_{n \in \mathbb{Z}}$ and $\left\{E_{n}\right\}_{n \in \mathbb{Z}}$ are two sequences of sets in $X$. The sequence of maps $\left\{f_{n}: D_{n} \rightarrow E_{n}\right\}_{n \in \mathbb{Z}}$ is said to be equi-continuous in $\left\{D_{n}\right\}_{n \in \mathbb{Z}}$ if for any $\varepsilon>0$, there exists $\delta>0$ such that $d\left(f_{i}^{n}(x), f_{i}^{n}(y)\right)<\varepsilon$ for all $n, i \in \mathbb{Z}$ and for all $x, y \in D_{n}$ with $d(x, y)<\delta$.

Definition 1.4. The point $p \in X$ is an almost periodic point if for every neighborhood $U$ of $p$ there exists some $k \in \mathbb{N}$ such that

$$
\left\{f_{i}^{i+n}(p): n=1, \ldots, k\right\} \cap U \neq \emptyset \text { and }\left\{f_{i}^{i-n}(p): n=1, \ldots, k\right\} \cap U \neq \emptyset,
$$

for every $i \in \mathbb{Z}$. The set of almost periodic points is denoted by $\mathcal{A P}\left(\left\{f_{n}\right\}_{n \in \mathbb{Z}}\right)$.

We denote $\gamma(A)$ is the set of all connected components of $X$ which intersect $A$ is nonempty. The following, we shall show the relationships between the Shadowable points and totally disconnected property.

Theorem 1.1. Let $\left(X,\left\{f_{n}\right\}_{n \in \mathbb{Z}}\right)$ with $X$ is a compact metric space and assume that

$$
\gamma\left(\mathcal{S}\left(\left\{f_{n}\right\}_{n \in \mathbb{Z}}\right) \cap \mathcal{A} \mathcal{P}\left(\left\{f_{n}\right\}_{n \in \mathbb{Z}}\right)\right) \subset \mathcal{C}\left(\left\{f_{n}\right\}_{n \in \mathbb{Z}}\right) .
$$

Then

$$
\mathcal{S}\left(\left\{f_{n}\right\}_{n \in \mathbb{Z}}\right) \cap \mathcal{A P}\left(\left\{f_{n}\right\}_{n \in \mathbb{Z}}\right) \subset X^{\text {deg }} .
$$

Theorem 1.2. Let $\left(X,\left\{f_{n}\right\}_{n \in \mathbb{Z}}\right)$ with $X$ is a compact metric space and assume that $\left\{f_{n}\right\}_{n \in \mathbb{Z}}$ is equicontinuous. Then

$$
X^{\operatorname{deg}} \subset \mathcal{S}\left(\left\{f_{n}\right\}_{n \in \mathbb{Z}}\right)
$$

\section{Proof of the Main Theorem}

Let us denote by $B[p, \varepsilon]$ is a ball with centre $p \in X$ and radius $\varepsilon>0$. First, we need the following definition. Then we have the following proposition.

Proposition 2.1. Let $\left(X,\left\{f_{n}\right\}_{n \in \mathbb{Z}}\right)$ with $X$ is a compact metric space. For any

$$
p \in \mathcal{S}\left(i,\left\{f_{n}\right\}_{n \in \mathbb{Z}}\right) \cap \mathcal{A P}\left(\left\{f_{n}\right\}_{n \in \mathbb{Z}}\right),
$$

then for all $\varepsilon>0$, there exists the order subsequence $\left\{n_{k}\right\}_{k \in \mathbb{Z}} \subset \mathbb{Z}\left(n_{0}=0\right)$ such that there exists $y \in X$ satisfies

$$
g_{0}^{k}(y) \in B[p, \varepsilon], \quad \forall k \in \mathbb{Z},
$$

where $g_{k}=f_{i+\sum_{h=0}^{k} n_{h}} \circ \cdots \circ f_{i+\sum_{h=0}^{k-1} n_{h}+1}, k \in \mathbb{Z}^{*}$ and $g_{0}=i d$. 
Proof.. By given, we have $p \in \mathcal{S}\left(i,\left\{f_{n}\right\}_{n \in \mathbb{Z}}\right) \cap \mathcal{A P}\left(\left\{f_{n}\right\}_{n \in \mathbb{Z}}\right)$. Therefore, let $\varepsilon>0$ is arbitrary constant, there is a $\delta>0$ such that for all $\delta$-pseudo-orbits $\left\{x_{n}\right\}_{n \in \mathbb{Z}}$ with $x_{i}=p$ is $\varepsilon$-shadowable. Due to $p \in \mathcal{A P}\left(\left\{f_{n}\right\}_{n \in \mathbb{Z}}\right)$, there is a number $k_{1} \geqslant 1$ such that $\left\{f_{i}^{i+n}(p): n=1, \ldots, k_{1}\right\} \cap U \neq \emptyset$ where $U=B[p, \delta / 2]$. It is implies that there is a number $n_{1}, i<n_{1} \leqslant i+k_{1}$ such that $d\left(f_{i}^{n_{1}}(p), p\right) \leqslant \delta / 2$. By $p \in \mathcal{A P}\left(\left\{f_{n}\right\}_{n \in \mathbb{Z}}\right)$ again, there is a number $k_{2} \geqslant 1$ such that $\left\{f_{i+n_{1}}^{i+n_{1}+n}(p): n=1, \ldots, k_{2}\right\} \cap B[p, \delta / 2] \neq \emptyset$. Then there is also a number $n_{2}, 0<n_{2} \leqslant k_{2}$ such that $d\left(f_{i+n_{1}}^{i+n_{1}+n_{2}}(p), p\right) \leqslant \delta / 2$. Repeat this procedure, we obtain that there is a sequence $\left\{n_{k}\right\}_{k \in \mathbb{Z}}\left(n_{0}=0\right)$ such that

$$
d\left(f_{i+n_{1}+\cdots+n_{k}}^{i+n_{1}+\cdots+n_{k+1}}(p), p\right) \leqslant \frac{\delta}{2}, \quad \forall k \in \mathbb{Z} .
$$

We choose the sequence in $X$, namely $\left\{x_{n}\right\}_{n \in \mathbb{Z}}$ which defined by

$$
x_{n}=\left\{\begin{array}{c}
f_{i+n_{1}+\cdots+n_{k}}^{i+n_{1}+\cdots+n_{k}+h}(p), \text { for } n=i+n_{1}+\cdots+n_{k}+h \text { and } h=1,2, \ldots, n_{k+1}-1 \\
p,
\end{array}\right.
$$

Then $\left\{x_{n}\right\}_{n \in \mathbb{Z}}$ is $\delta$-pseudo-orbit. Indeed,

Case 1. If $n=i+n_{1}+\cdots+n_{k}+h$ with $h=0,2, \ldots, n_{k+1}-2$. Then

$$
d\left(f_{n}\left(x_{n}\right), x_{n+1}\right)=d\left(f_{i+n_{1}+\cdots+n_{k}+h}\left(f_{i+n_{1}+\cdots+n_{k}}^{i+n_{1}+\cdots+n_{k}+h}(p)\right), f_{i+n_{1}+\cdots+n_{k}}^{i+n_{1}+\cdots+n_{k}+h+1}(p)\right)=0 .
$$

Case 2. If $n=i+n_{1}+\cdots+n_{k+1}-1$. Then we have the following estimation

$$
\begin{aligned}
d\left(f_{n}\left(x_{n}\right), x_{n+1}\right) & =d\left(f_{i+n_{1}+\cdots+n_{k+1}-1}\left(f_{i+n_{1}+\cdots+n_{k}}^{i+n_{1}+\cdots+n_{k+1}-1}(p)\right), p\right) \\
& =d\left(f_{i+n_{1}+\cdots+n_{k}}^{i+n_{1}+\cdots+n_{k+1}}(p), p\right) \\
& \leqslant \delta / 2 .
\end{aligned}
$$

It is clear that $x_{i}=p$. Hence, $\left\{x_{n}\right\}_{n \in \mathbb{Z}}$ is $\delta$-pseudo-orbit through $p$ at the time $i$. Therefore, there is $y \in X$ such that $d\left(f_{i}^{n}(y), x_{n}\right) \leqslant \varepsilon$ for all $n \in \mathbb{Z}$. Particularly, when $n=i+n_{1}+\cdots+n_{k}$ we have

$$
d\left(f_{i}^{i+n_{1}+\cdots+n_{k}}(y), x_{i+n_{1}+\cdots+n_{k}}\right) \leqslant \varepsilon, \quad \forall k \in \mathbb{Z} .
$$

Set $g_{k}=f_{i+\sum_{h=0}^{k} n_{h}} \circ \cdots \circ f_{i+\sum_{h=0}^{k-1} n_{h}+1} k \in \mathbb{Z}^{*}$ and $g_{0}=i d$. From the last inequality we have for every $k \in \mathbb{Z}$ then

$$
d\left(g_{0}^{k}(y), p\right) \leqslant \varepsilon
$$

This proposition is proved.

Proposition 2.2. Let $p \in \mathcal{S}\left(i,\left\{f_{n}\right\}_{n \in \mathbb{Z}}\right)$, then for every $\varepsilon>0$, there is $\delta$ such that for all $\left\{x_{n}\right\}_{n \in \mathbb{Z}} i s$ $\delta$-pseudo-orbit with $x_{i} \in B[p, \delta]$ then $\left\{x_{n}\right\}_{n \in \mathbb{Z}}$ is $\varepsilon$-shadowed.

Proof.. Suppose by contradiction that there are $\varepsilon>0$ and a sequence of $\frac{1}{k}$-pseudo-orbit $\left\{x^{k}\right\}_{k \in \mathbb{N}^{+}}$with $x_{i}^{k} \in B\left[p, \frac{1}{k}\right]$ which cannot be $2 \varepsilon$-shadowed for all $k \in \mathbb{N}^{+}$. By hypothesis, for this $\varepsilon$, we take $\delta<\varepsilon$ in terms of the shadowable point $p$. As $X$ is compact, $f_{i}$ is uniformly continuous so we can fix $k$ large such that $\max \left\{d\left(f_{i}(p), f_{i}\left(x_{i}^{k}\right)\right), \frac{1}{k}\right\} \leqslant \frac{\delta}{2}$. Once we fix this $k$, we define the sequence $\left\{\hat{x}_{n}\right\}_{n \in \mathbb{Z}}$ by

$$
\hat{x}_{n}= \begin{cases}x_{n}^{k}, & \text { if } n \neq i \\ p, & \text { if } n=i\end{cases}
$$

Clearly $\left.d\left(f_{n}(\hat{(} x)_{n}\right), x_{n+1}\right) \leqslant \frac{1}{k} \leqslant \delta$ for $n \neq i-1, i$. Since

$$
d\left(f_{i-1}\left(\hat{x}_{i-1}\right), \hat{x}_{i}\right)=d\left(f_{i-1}\left(x_{i-1}^{k}\right), p\right) \leqslant d\left(f_{i-1}\left(x_{i-1}^{k}\right), x_{i}^{k}\right)+d\left(x_{i}^{k}, p\right) \leqslant \frac{1}{k}+\frac{1}{k} \leqslant \delta
$$

and

$$
d\left(f_{i}\left(\hat{x}_{i}\right), \hat{x}_{i+1}\right)=d\left(f_{i}(p), x_{i+1}^{k}\right) \leqslant d\left(f_{i}(p), f_{i}\left(x_{i}^{k}\right)\right)+d\left(f_{i}\left(x_{i}^{k}\right), x_{i+1}^{k}\right) \leqslant \frac{\delta}{2}+\frac{1}{k} \leqslant \delta .
$$


It implies that $\left\{\hat{x}_{n}\right\}_{n \in \mathbb{Z}}$ is a $\delta$-pseudo-orbit. On the other hand, $\hat{x}_{i}=p$, by definition we obtain that $\left\{\hat{x}_{n}\right\}_{n \in \mathbb{Z}}$ is $\varepsilon$-shadowed, namely, there is $y \in X$ such that $d\left(f_{i}^{n}(y), \hat{x}_{n}\right) \leqslant \varepsilon$ for every $n \in \mathbb{Z}$.

Clearly $d\left(f_{i}^{n}(y), x_{n}^{k}\right)=d\left(f_{i}^{n}(y), \hat{x}_{n}\right) \leqslant 2 \varepsilon$ for $n \neq i$. For $n=i$ we obtain

$$
d\left(f_{i}^{n}(y), x_{n}^{k}\right)=d\left(y, x_{i}^{k}\right) \leqslant d(y, p)+d\left(p, x_{i}^{k}\right) \leqslant \varepsilon+\frac{1}{k} \leqslant 2 \varepsilon .
$$

It follows that $\left\{x_{n}^{k}\right\}_{n \in \mathbb{Z}}$ is $2 \varepsilon$-shadowed, that is absurd. This contradiction proves the result.

\section{Proof of the Theorem 1.1.}

We assume contrary that there is a $p$ which belongs to $\mathcal{S}\left(\left\{f_{n}\right\}_{n \in \mathbb{Z}}\right) \cap \mathcal{A P}\left(\left\{f_{n}\right\}_{n \in \mathbb{Z}}\right)$ but does not in $X^{\text {deg }}$. Then $\operatorname{diam} \gamma(\{p\})>0$ and hence choose $\varepsilon>0$ such that $0<11 \varepsilon<\operatorname{diam} \gamma(p)$. By given, we have $p \in \mathcal{S}\left(i_{0},\left\{f_{n}\right\}_{n \in \mathbb{Z}}\right) \cap \mathcal{A P}\left(\left\{f_{n}\right\}_{n \in \mathbb{Z}}\right)$ for some $i_{0} \in \mathbb{Z}$. From Proposition 2.1 and Proposition 2.2 , with $\delta$ $(\delta<\varepsilon)$ of pseudo-orbit of $\left\{f_{n}\right\}_{n \in \mathbb{Z}}$ respect to $\varepsilon$ above, there is the order subsequence $\left\{n_{k}\right\}_{k \in \mathbb{Z}} \subset \mathbb{Z}, n_{0}=0$

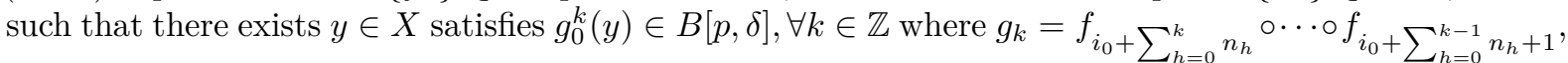
$k \in \mathbb{Z}^{*}$ and $g_{0}=i d$, moreover, every $\delta$-pseudo-orbit through $y$ at time $i$ is also $\varepsilon$-shadowed. It is easy to check that $p$ also belongs to $\mathcal{S}\left(0,\left\{g_{k}\right\}_{k \in \mathbb{Z}}\right)$. Indeed, let $\left\{x_{n}\right\}_{n \in \mathbb{Z}}$ is $\delta$-pseudo-orbit of $\left\{g_{k}\right\}_{k \in \mathbb{Z}}$, we define

$$
\beta_{n}= \begin{cases}f_{n_{k}}^{n_{k}+i}\left(x_{n_{k}}\right), & \text { if } n=n_{k}+i, i=1, \ldots, n_{k+1}-1 \\ x_{n_{k}}, & \text { if } n=n_{k} .\end{cases}
$$

Then, $\left\{\beta_{n}\right\}_{n \in \mathbb{Z}}$ is $\delta$-pseudo-orbit of $\left\{f_{n}\right\}_{n \in \mathbb{Z}}$ which has $\beta_{i_{0}}=p$. Therefore there is $x \in X$ such that

$$
d\left(f_{i_{0}}^{n}(x), \beta_{n}\right) \leqslant \varepsilon, \quad, \forall n \in \mathbb{Z} .
$$

It mean that $d\left(g_{0}^{k}(x), x_{n}\right) \leqslant \varepsilon, \forall k \in \mathbb{Z}$. Hence, $p \in \mathcal{S}\left(0,\left\{g_{k}\right\}_{k \in \mathbb{Z}}\right)$, in other word, every $\delta$-pseudo-orbits of $\left\{g_{n}\right\}_{n \in \mathbb{Z}}$ through $p$ at time 0 is $\varepsilon$-shadowed. Similarly, we also have every $\delta$-pseudo-orbits of $\left\{g_{n}\right\}_{n \in \mathbb{Z}}$ through $y$ at time 0 is $\varepsilon$-shadowed. Next, since $\gamma(\{p\})$ is compact, connected and by (1), we could choose the finite sequence, namely $\left\{q_{i}\right\}_{i=0}^{n_{0}}$, which satisfies

i) $q_{0}=y, q_{i} \in \gamma(\{p\}) \cap \mathcal{C}\left(\left\{f_{n}\right\}_{n \in \mathbb{Z}}\right), \forall i=1, \ldots, n_{0}$,

ii) $d\left(q_{i}, q_{i+1}\right) \leqslant \delta / 2, i=0,1, \ldots, n_{0}$ and $d\left(q_{n_{0}}, q_{0}\right) \leqslant \delta / 2$,

iii) $\gamma(\{p\})=\bigcup_{i=1}^{n_{0}} B\left[q_{i}, \delta\right]$.

Due to (i), there is a number $m_{i}$ (large enough) such that $d\left(g_{m_{i-1}}^{m_{i}}\left(q_{i}\right), q_{i}\right) \leqslant \delta / 2$ for all $i=0,1,2, \ldots, n_{0}$ $\left(m_{0}=1\right)$. We can choose sequence $\left\{m_{i}\right\}_{0}^{n_{0}}$ such that $1=m_{0}<m_{1}<m_{2}<\cdots<m_{n_{0}}$. Then we have following claims.

Claim 1. The sequence

$$
\xi_{n}= \begin{cases}g_{0}^{n}\left(q_{0}\right), & \text { if } n \leqslant 0 \\ g_{m_{i}}^{n}\left(q_{i+1}\right), & \text { if } m_{i} \leqslant n<m_{i+1}, i=0,1,2, \ldots, m_{n_{0}} \\ g_{m_{n_{0}}}^{n}\left(q_{0}\right), & \text { if } n \geqslant m_{n_{0}} .\end{cases}
$$

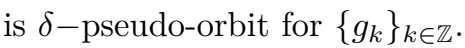

Indeed, to order this claim one divide into several cases.

Case 1. If $n \neq 0, n \neq m_{i}-1, i=1, \ldots, n_{0}$ then

$$
d\left(g_{n}\left(\xi_{n}\right), \xi_{n+1}\right)= \begin{cases}d\left(g_{n}\left(g_{0}^{n}\left(q_{0}\right)\right), g_{0}^{n+1}\left(q_{0}\right)\right), & \text { if } n<0 \\ d\left(g_{n}\left(g_{m_{i}}^{n}\left(q_{i+1}\right)\right), g_{m_{i}}^{n+1}\left(q_{i+1}\right)\right), & \text { if } m_{i} \leqslant n<m_{i+1}-1, i=0,1,2, \ldots, m_{n_{0}} \\ d\left(g_{n}\left(g_{m_{n_{0}}}^{n}\left(q_{0}\right)\right), g_{m_{n_{0}}}^{n+1}\left(q_{0}\right)\right), & \text { if } n \geqslant m_{n_{0}} .\end{cases}
$$

We obtain $d\left(g_{n}\left(\xi_{n}\right), \xi_{n+1}\right)=0$ in this case.

Case 2. If $n=0$, then

$$
d\left(g_{0}\left(\xi_{0}\right), \xi_{1}\right)=d\left(q_{0}, q_{1}\right) \leqslant \delta / 2 \leqslant \delta .
$$


Case 3. If $n=m_{i}-1\left(i=1,2, \ldots, n_{0}-1\right)$ then

$$
\begin{aligned}
d\left(g_{n}\left(\xi_{n}\right), \xi_{n+1}\right) & =d\left(g_{m_{i}-1}\left(g_{m_{i-1}}^{m_{i}-1}\left(q_{i}\right)\right), q_{i+1}\right) \\
& =d\left(g_{m_{i-1}}^{m_{i}}\left(q_{i}\right), q_{i+1}\right) \\
& \leqslant d\left(g_{m_{i-1}}^{m_{i}}\left(q_{i}\right), q_{i}\right)+d\left(q_{i}, q_{i+1}\right) \\
& \leqslant \delta / 2+\delta / 2=\delta .
\end{aligned}
$$

Case 4. If $n=m_{n_{0}}-1$ then

$$
\begin{aligned}
d\left(g_{n}\left(\xi_{n}\right), \xi_{n+1}\right) & =d\left(g_{m_{n_{0}}-1}\left(g_{m_{n_{0}-1}}^{m_{n_{0}}-1}\left(q_{n_{0}}\right)\right), q_{0}\right) \\
& =d\left(g_{m_{i-1}}^{m_{i}}\left(q_{i}\right), q_{i+1}\right) \\
& \leqslant d\left(g_{m_{i-1}}^{m_{i}}\left(q_{i}\right), q_{n_{0}}\right)+d\left(q_{n_{0}}, q_{0}\right) \\
& \leqslant \delta / 2+\delta / 2=\delta .
\end{aligned}
$$

Hence, the claim is proved.

On the other hand, $\left\{\xi_{n}\right\}_{n \in \mathbb{Z}}$ through $y$ at the time 0 and $y \in B[p, \delta]$, from Proposition 2.2, there exists $\hat{y} \in X$ such that

$$
d\left(g_{0}^{n}(\hat{y}), \xi_{n}\right) \leqslant \varepsilon, \quad \forall n \in \mathbb{Z} .
$$

In particular, there are finite sequence $\left\{l_{i}\right\}_{i=0}^{n_{0}}$ such that

$$
d\left(g_{0}^{l_{i}}(\hat{y}), q_{i}\right) \leqslant \varepsilon, \quad i=1,2, \ldots, n_{0} .
$$

Claim 2. For every $z \in \gamma(\{p\})$ then $d(z, p) \leqslant 5 \varepsilon$.

In fact, let any $z \in \gamma(\{p\})=\bigcup_{i=1}^{n_{0}} B\left[q_{i}, \delta\right]$. Then there is $i=0,1, \ldots, n_{0}$ such that $d\left(z, q_{i}\right) \leqslant \delta$. Therefore,

$$
\begin{aligned}
d\left(g_{0}^{l_{i}}(\hat{y}), z\right) & \leqslant d\left(g_{0}^{l_{i}}(\hat{y}), q_{i}\right)+d\left(q_{i}, z\right) \\
& \leqslant \varepsilon+\delta \leqslant 2 \varepsilon .
\end{aligned}
$$

To prove this claim, we divide into 2 cases of $l_{i}$.

Case 1. $m_{1} \leqslant l_{i} \leqslant m_{n_{0}}-1$.

Since $(1)$, we have $g_{0}^{l_{i}}(\hat{y}) \in \omega\left(g_{0}^{l_{i}}(\hat{y})\right)$. It implies there is $i_{0}>m_{n_{0}}$ such that

$$
d\left(g_{0}^{i_{0}}(\hat{y}), g_{0}^{l_{i}}(\hat{y})\right) \leqslant \varepsilon .
$$

In addition, due to $i_{0}>m_{n_{0}}$, we have $\xi_{i_{0}}=g_{0}^{h}(y)$ for some $h \in \mathbb{Z}$. Further, $g_{0}^{k}(y) \in B[p, \delta]$, we obtain

$$
d\left(g_{0}^{i_{0}}(\hat{y}), p\right) \leqslant d\left(g_{0}^{i_{0}}(\hat{y}), \xi_{i_{0}}\right)+d\left(\xi_{i_{0}}, p\right) \leqslant 2 \varepsilon .
$$

Hence,

$$
\begin{aligned}
d(z, p) & \leqslant d\left(z, g_{0}^{l_{i}}(\hat{y})\right)+d\left(g_{0}^{l_{i}}(\hat{y}), d\left(z, g_{0}^{i_{0}}(\hat{y})\right)\right)+d\left(z, g_{0}^{i_{0}}(\hat{y}), z\right) \\
& \leqslant 2 \varepsilon+\varepsilon+2 \varepsilon=5 \varepsilon .
\end{aligned}
$$

Case 2. $l_{i} \leqslant m_{1}$ or $l_{i} \geqslant m_{n_{0}}-1$.

It is clear to see that $\xi_{l_{i}}=g_{0}^{h}(y)$ for some $h \in \mathbb{Z}$, it implies

$$
d\left(\xi_{l_{i}}, p\right)=d\left(g_{0}^{h}(y), p\right) \leqslant \delta \leqslant \varepsilon .
$$

Hence,

$$
\begin{gathered}
d\left(g_{0}^{l_{i}}(\hat{y}), p\right) \leqslant d\left(g_{0}^{l_{i}}(\hat{y}), \xi_{l_{i}}\right)+d\left(\xi_{l_{i}}, p\right) \\
\varepsilon+\varepsilon=2 \varepsilon .
\end{gathered}
$$

Therefore,

$$
\begin{gathered}
d(z, p) \leqslant d\left(z, g_{0}^{l_{i}}(\hat{y})\right)+d\left(g_{0}^{l_{i}}(\hat{y}), p\right) \\
2 \varepsilon+2 \varepsilon<5 \varepsilon .
\end{gathered}
$$

This proves this claim. 
From the Claim 2, for every $u, v \in \gamma(\{p\})$ then

$$
d(u, v) \leqslant d(u, p)+d(p, v) \leqslant 5 \varepsilon+5 \varepsilon=10 \varepsilon .
$$

This contradicts the choice of $\varepsilon$. The proof is complete.

\section{Proof of the Theorem 1.2.}

We take $p \in X^{\text {deg }}$ and $\varepsilon>0$. Since $\left\{f_{n}\right\}_{n \in \mathbb{Z}}$ is equi-continuous, there exists $\varepsilon^{\prime}>0$ such that $d\left(f_{i}^{n}(x), f_{i}^{n}(y)\right) \leqslant \varepsilon(\forall i, n \in \mathbb{Z})$, for all $x, y \in X, d(x, y) \leqslant \varepsilon^{\prime}$. By Proposition 3.1.7 in [11], there is a open and closed subset $U$ of $X$ such that

i) $p \in U$ and $\operatorname{diam}(U) \leqslant \varepsilon^{\prime}$,

ii) $\operatorname{dist}(U, X \backslash U)>2 \alpha>0$ for some $A \in \mathbb{R}^{+}$.

By the equi-continuous property of $\left\{f_{n}\right\}_{n \in \mathbb{Z}}$ again, we can choose $\delta>0$ such that $x, y \in X$ and $d(x, y)<\delta$ then $d\left(f_{i}^{n}(x), f_{i}^{n}(y)\right) \leqslant \alpha(\forall i, n \in \mathbb{Z})$. Now take a $\delta$-pseudo-orbits $\left\{x_{n}\right\}_{n \in \mathbb{Z}}$ with $x_{i}=p$ for some $i \in \mathbb{Z}$. From definition $d\left(f_{i}\left(x_{i}\right), x_{i+1}\right) \leqslant \delta$, it implies

$$
d\left(f_{i+1}^{i} f_{i}\left(x_{i}\right), f_{i+1}^{i}\left(x_{i+1}\right)\right) \leqslant \alpha \Leftrightarrow d\left(p, f_{i}^{-1}\left(x_{i+1}\right)\right) \leqslant \alpha .
$$

If $f_{i}^{-1}\left(x_{i+1}\right) \in X \backslash U$, from i) and ii), we have

$$
2 \alpha<\operatorname{dist}(X \backslash U, U) \leqslant d\left(p, f_{i}^{-1}\left(x_{i+1}\right)\right) \leqslant \alpha,
$$

which is contradiction. Then $f_{i}^{-1}\left(x_{i+1}\right) \in U$. Next, since $d\left(f_{i+1}\left(x_{i+1}\right), x_{i+2}\right) \leqslant \delta$ so

$$
d\left(f_{i+2}^{i} f_{i+1}\left(x_{i+1}\right), f_{i+2}^{i}\left(x_{i+2}\right)\right) \leqslant \alpha \Leftrightarrow d\left(f_{i}^{-1}\left(x_{i+1}\right), f_{i+2}^{i}\left(x_{i+2}\right)\right) \leqslant \alpha .
$$

If $f_{i+2}^{i}\left(x_{i+2}\right) \in X \backslash U$, from $\left.\mathbf{i}\right)$ and $\mathbf{i i}$ ) again, it implies

$$
2 \alpha<\operatorname{dist}(X \backslash U, U) \leqslant d\left(f_{i}^{-1}\left(x_{i+1}\right), f_{i+2}^{i}\left(x_{i+2}\right)\right) \leqslant \alpha,
$$

which is absurd. Hence, $f_{i+2}^{i}\left(x_{i+2}\right) \in U$. Repeating the argument we obtain $f_{i+n}^{i}\left(x_{i+n}\right) \in U$ for every $n \in \mathbb{Z}$. It follows that $d\left(p, f_{i+n}^{i}\left(x_{i+n}\right)\right) \leqslant \varepsilon^{\prime}$. Therefore,

$$
d\left(f_{i}^{i+n}(p), f_{i}^{i+n} f_{i+n}^{i}\left(x_{i+n}\right)\right) \leqslant \varepsilon \Leftrightarrow d\left(f_{i}^{i+n}(p), x_{i+n}\right) \leqslant \varepsilon, \quad \forall n \in \mathbb{Z} .
$$

It means that $p \in \mathcal{S}\left(\left\{f_{n}\right\}_{n \in \mathbb{Z}}\right)$, which is our claim.

\section{Conclusion}

In this paper, we extend the concept of the shadowable point in case of non-autonomous dynamical systems and investigate the relations between the set of shadowable points and totally disconnected property. The results are also related to almost periodic points and the points with converging semiorbits.

Acknowledgments. The first author was supported in part by the VNU Project of Vietnam National University No. QG15.01.

\section{References}

1. N. Aoki and K. Hiraide, "Topological Theory of Dynamical Systems. Recent Advances," North-Holland Mathematical Library, vol. 52, 1994.

2. S. Y. Pilyugin, "Shadowing in Dynamical Systems. Lecture Notes in Mathematics," Springer, Berlin, vol. 1706, 1999.

3. G. C. Yuan and J. A. Yorke, "An open set of maps for which every point is absolutely nonshadowable," Proc. Am. Math. Soc., vol. 128, no. 3, pp. 909-918, 2000.

4. N. Kawaguchi, "Properties of Shadowable Points: Chaos and Equicontinuity," Bull. Braz. Math. Soc., vol. 48, no. 4, pp. 599-622, 2017. 
5. P. Duarte and S. Klein, "Lyapunov Exponents of Linear Cocycles," Atlantis press, 2016.

6. C. A. Morales, "Shadowable points," Dyn. Syst., vol. 31, no. 3, pp. 347-356, 2016.

7. T. Eirola, O. Nevanlinna and S. Pilyugin, "Limit shadowing property," Numer. Funct. Anal. Optim., vol. 18, no. 1-2, pp. 75-92, 1997.

8. B. Carvalho and W. Cordeiro, "N-expansive homeomorphisms with the shadowing property," J. Differential Equation, vol. 261, no. 6, pp. 3734-3755, 2016.

9. W. Reddy, "Pointwise expansion homeomorphism," J. London Math. Soc., vol. s2-2, no. 2, 232-236, 1970.

10. F. Balibrea, T. Downarowicz and R. Hric, "Almost totally disconnected minimal systems," Ergodic Theory Dyn Syst., vol. 29, no. 3, pp. 737-766, 2009.

11. A. Arhangel'skii, M. Tkachenko, "Topological groups and related structures," Atlantis Studies in Mathematics, 1. Atlantis Press, Paris; World Scientifc Publishing Co. Pte. Ltd., Hackensack, NJ, 2008. 